University of Montana

ScholarWorks at University of Montana

$5-8-2007$

\title{
Microwave Absorption of Patterned Arrays of Nanosized Magnetic Stripes with Different Aspect Ratios
}

\author{
Leszek M. Malkinski \\ University of New Orleans \\ Minghui Yu \\ University of New Orleans \\ Andriy Vovk \\ University of New Orleans, avovk@uno.edu \\ Donald J. Scherer II \\ University of New Orleans \\ Leonard Spinu \\ University of New Orleans \\ See next page for additional authors \\ Follow this and additional works at: https://scholarworks.umt.edu/chem_pubs \\ Part of the Biochemistry Commons, Chemistry Commons, and the Physics Commons \\ Let us know how access to this document benefits you.
}

\section{Recommended Citation}

Malkinski, Leszek M.; Yu, Minghui; Vovk, Andriy; Scherer, Donald J. II; Spinu, Leonard; Zhou, Weilie; Whittenburg, Scott L.; Davis, Zachary; and Jung, Jin-Seung, "Microwave Absorption of Patterned Arrays of Nanosized Magnetic Stripes with Different Aspect Ratios" (2007). Chemistry and Biochemistry Faculty Publications. 70.

https://scholarworks.umt.edu/chem_pubs/70

This Article is brought to you for free and open access by the Chemistry and Biochemistry at ScholarWorks at University of Montana. It has been accepted for inclusion in Chemistry and Biochemistry Faculty Publications by an authorized administrator of ScholarWorks at University of Montana. For more information, please contact scholarworks@mso.umt.edu. 


\section{Authors}

Leszek M. Malkinski, Minghui Yu, Andriy Vovk, Donald J. Scherer II, Leonard Spinu, Weilie Zhou, Scott L. Whittenburg, Zachary Davis, and Jin-Seung Jung 


\title{
Microwave absorption of patterned arrays of nanosized magnetic stripes with different aspect ratios
}

\author{
Leszek M. Malkinski, ${ }^{a}$ Minghui Yu, Andriy Y. Vovk, Donald J. Scherer II, Leonard Spinu, \\ Weilie Zhou, Scott Whittenburg, and Zachary Davis \\ Advanced Materials Research Institute, University of New Orleans, New Orleans, Louisiana 70148 \\ Jin-Seung Jung \\ Department of Chemistry, Kangnung National University, Kangnung 210-702, South, Korea
}

(Presented on 9 January 2007; received 3 November 2006; accepted 27 December 2006; published online 8 May 2007)

\begin{abstract}
Arrays consisting of nanosized stripes of Permalloy with different length-to-width ratios have been fabricated using electron beam nanolithography, magnetron sputtering, and lift-off process. These stripes have a thickness of $100 \mathrm{~nm}$, a width of $300 \mathrm{~nm}$, and different lengths ranging from $300 \mathrm{~nm}$ to $100 \mu \mathrm{m}$. The stripes are separated by a distance of $1 \mu \mathrm{m}$. Magnetization hysteresis loops were measured using a superconducting quantum interference device susceptometer. Microwave absorption at $9.8 \mathrm{GHz}$ was determined by means of ferromagnetic resonance technique. The dependence of the resonant field on the angle between the nanostructure and the in-plane dc magnetic field indicates the presence of uniaxial magnetic anisotropy associated with the aspect ratio of the stripes. A maximum change of the resonant field of 1600 Oe was observed in the longest stripes, yet it was only 200 Oe for square shaped stripes. The linewidth of the resonant curve varied with the angle, in the range from 120 to 300 Oe. Most of the ferromagnetic resonance spectra exhibited multiple resonant peaks due to dimensional confinement of spin waves in the nanosized stripes. The maximum squareness of the magnetization hysteresis loop was for the field applied along the stripes, but the coercivity did not have a monotonic angular dependence as expected from the Stoner-Wohlfarth model for coherent magnetization rotation of the systems with uniaxial anisotropy. (C) 2007 American Institute of Physics. [DOI: 10.1063/1.2712820]
\end{abstract}

\section{INTRODUCTION}

Research on magnetization processes and spin dynamics in magnetic nanoparticles is important because of their unique magnetic properties and potential applications in information technology, wireless communications, and medicine. Arrays of nanoparticles with large aspect ratios can be produced by various methods, ${ }^{1-3}$ but electron beam nanolithography seems to be the most flexible of all in defining the lateral structures. Arbitrary shapes with dimensions in the range from $30 \mathrm{~nm}$ to $100 \mu \mathrm{m}$ can be patterned using this method. The frequency of operation of computers and cellphones tends to increase in order to enhance the efficiency of data processing and broaden the frequency band for wireless telecommunications. Currently, these frequencies are approaching the microwave region. Conventional magnetic microwave materials, such as spinels, hexaferrites, and garnets, are insulators, and metals were usually excluded from the microwave applications because conduction losses were too large in these materials.

This restriction does not apply to nanostructures which are much smaller than the penetration depth of microwaves. The advantages of the nanostructures of ferromagnetic transition metals over conventional metals and alloys are a larger magnetization and a simpler technology which can easily be

\footnotetext{
${ }^{\text {a) }}$ Author to whom correspondence should be addressed; FAX: (504) 2801346; electronic mail: lmalkins@uno.edu
}

integrated with the common processes in semiconductor technology. Different applications of ferromagnetic metals in tunable microwave filters have recently been developed. ${ }^{4-7}$ The aim of this work is to investigate the effect of shape anisotropy on microwave absorption, which can be used to tailor microwave spectra by designing arrays consisting of stripes or wires with different aspect ratios.

\section{EXPERIMENT}

Fabrication of the arrays was performed using the method of electron beam nanolithography. Spin coating techniques were used to cover the $\mathrm{Si}(100)$ substrates with a thin layer of polymethyl methacrylate (PMMA). The patterns of arrays were defined by electron beam writing on the PMMA resin using a LEO $1530 \mathrm{VP}$ field emission scanning electron microscope (FESEM) operating at $30 \mathrm{eV}$. The patterned area of $1.65 \times 1.65 \mathrm{~mm}^{2}$ was subdivided into an array of 15 $\times 15$ identical patches with dimensions of $100 \times 100 \mu \mathrm{m}^{2}$ each. Each patch had the form of an array of nanosized stripes with a width of $300 \mathrm{~nm}$ separated by a distance of $1 \mu \mathrm{m}$. Permalloy film with nominal composition of $81 \% \mathrm{Ni}$ and $19 \% \mathrm{Fe}$ and thickness of $100 \mathrm{~nm}$ was deposited onto the resin by magnetron sputtering at the rate of $0.2 \mathrm{~nm} / \mathrm{s}$ at an Ar pressure of 3 mTorr. The thickness of the films was measured using a crystal growth monitor and the thickness of the stripes was verified by measuring surface profiles of the array by means of the atomic force. Final structures were made by a lift-off process, consisting in the removal of the resin 


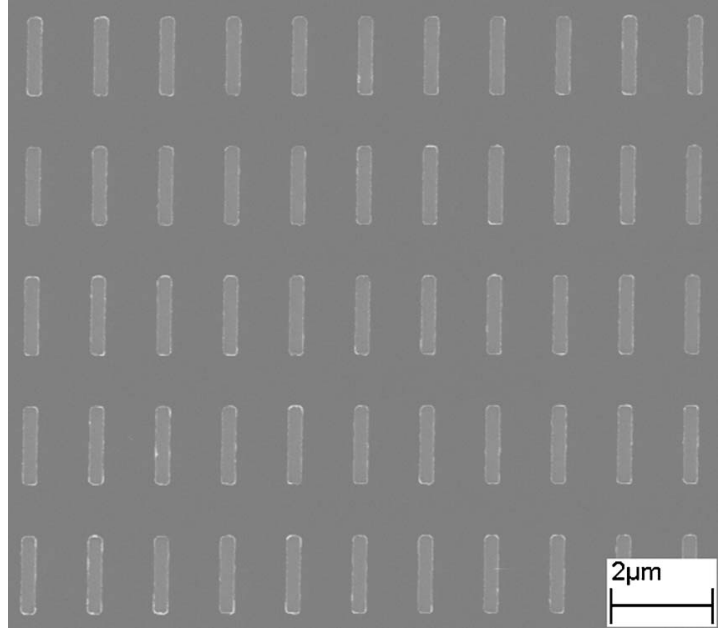

FIG. 1. FESEM image of a small fraction of the array of the weakly interacting Permalloy stripes with the thickness of $100 \mathrm{~nm}$ and 1:5 width to length ratio.

and the overlaying film in an ultrasonic bath of acetone. A series of six samples consisting of stripes with lengths of $300 \mathrm{~nm}, 600 \mathrm{~nm}, 900 \mathrm{~nm}, 1.5 \mu \mathrm{m}, 3 \mu \mathrm{m}$, and $100 \mu \mathrm{m}$ were fabricated, corresponding to the aspect ratios of 1:1, 1:2, 1:3, $1: 5,1: 10$, and $1: 333$ of the width to length. The quality of each pattern was tested using FESEM and atomic force microscopy (AFM). A representative FESEM image of an array of magnetic nanosized stripes with the aspect ratio of 1:5 is shown in Fig. 1. Magnetic hysteresis loops were measured at room temperature using an MPMS XL superconducting quantum interference device (SQUID) susceptometer from Quantum Design. Microwave absorption of the arrays was studied at room temperature by means of a ferromagnetic resonance technique using an X-band Bruker EMX300 electron paramagnetic resonance (EPR) spectrometer.

\section{RESULTS AND DISCUSSION}

Magneatization curves have been measured with the magnetic field applied in the plane of the arrays. Representative magnetization hysteresis loops measured for three different angles between the field and the long axis of the $100 \mu \mathrm{m}$ long stripes, i.e., $0^{\circ}, 45^{\circ}$, and $90^{\circ}$, are displayed in Fig. 2. The squareness of the loop is largest for the field orientation along the stripes, as expected for an object with uniaxial anisotropy, and it constantly decreases when the field rotates out of the easy anisotropy axis. However, the behavior of the coercivity differs from that predicted by the Stoner-Wohlfarth model for coherent magnetization rotation. The coercivity for the angle of $45^{\circ}$ is larger than for the field along the easy or hard anisotropy axis. This kind of angular dependence was observed in the $\mathrm{Ni}$ nanowires grown electrochemically in porous alumina templates. ${ }^{7}$ The possible mechanism of magnetization reversal which leads to reduced coercivity involves domain nucleation at the ends of the wires and propagation of the wall along the wires towards the opposite end. ${ }^{8,9}$

Since the quasistatic magnetization processes in the nanosized stripes seem to be rather complex, the question that arises is whether the shape anisotropy is significant for

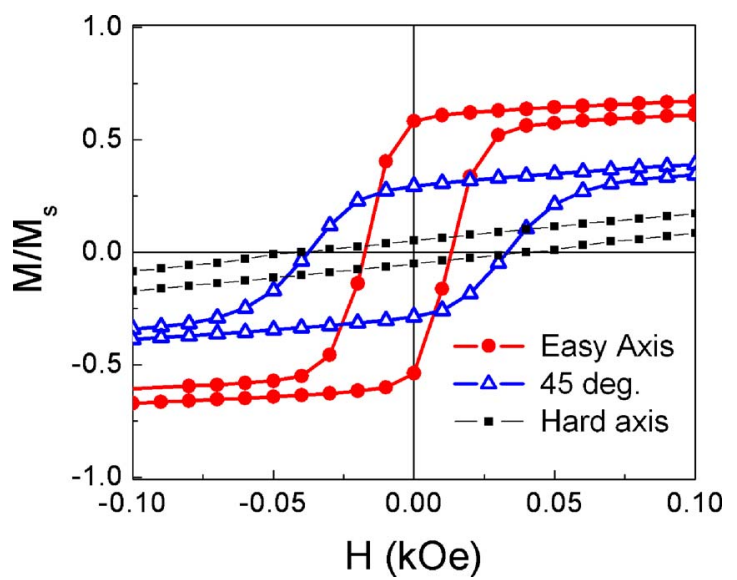

FIG. 2. (Color online) Magnetization hysteresis loops of the array of $100 \mu \mathrm{m}$ long stripes measured with the applied field in the plane of the array and at three different angles between the field and the structure: along the stripes (easy magnetization axis), $45^{\circ}$ with respect to the stripes, and across the stripes (hard magnetization axis).

the dynamic response of the arrays. At the microwave frequency of $9.8 \mathrm{GHz}$ of the resonant cavity, the ferromagnetic resonance in the Permalloy stripes occurs at fields above 1 kOe. Hysteresis loops in Fig. 2 indicate that at this magnitude of the field the nanosized stripes are already magnetically saturated, thus the stripes are single domains. Because of the large spacing $(1 \mu \mathrm{m})$ between the nanosized stripes, the interactions among them are supposed to be weak, and consequently, the microwave response of the array is expected to reflect the properties of individual stripes. The results of the measurements of the microwave absorption are summarized in Fig. 3. This figure shows the angular dependencies of the resonant fields for five arrays of stripes with different aspect ratios. The results for the stripes with the aspect ratio of 1:10 almost completely overlapped with the curve representing the longest stripes (ratio 1:333), and for clarity of the figure, they were omitted. The curves for all the samples, except for the square nanoparticles, display a single

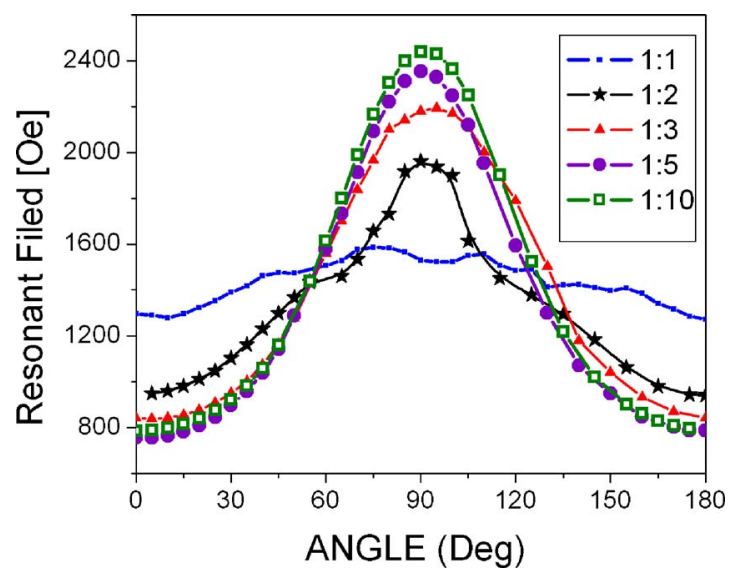

FIG. 3. (Color online) Dependencies of the resonant field on the angle between the long axis of the stripes and the in-plane applied field for the five patterned arrays of stripes with different aspect ratios. The smallest resonant fields occur for the direction of the field along the stripes, whereas maximum resonant fields were found for the transverse direction of the field. The difference between the smallest and the largest resonant fields, for a given array, is related to the magnitude of the shape anisotropy of the stripes. 
maximum for the direction of the in-plane magnetic field transverse to the stripes. This behavior of the resonant field is consistent with the presence of the uniaxial shape anisotropy of the stripes. The magnitude of this anisotropy is proportional to the range of the resonant field between the easy axis (i.e., at $0^{\circ}$ or for the field along the stripes) and the hard axis (i.e., at $90^{\circ}$ or for the field transverse to the stripes). In the absence of magnetocrystalline anisotropy, the relation among the frequency $f_{r}$ of the resonant absorption, the applied field $H$, and the uniaxial anisotropy field $H_{U}$ is given by the formula [in Systeme International (SI) units]

$$
f_{r}=\left(\frac{\gamma}{2 \pi}\right) \sqrt{\left(H+H_{U}\right)\left(H+H_{U}+M_{S}\right)},
$$

where $\gamma$ is the gyromagnetic ratio and $M_{S}$ is the saturation magnetization of the ferromagnetic material.

Initially, the anisotropy increases very quickly with increasing aspect ratio (length/with) of the stripes, but it nearly saturates for the aspect ratios which are larger than 10 . The maximum range of the angular variation of the resonant field reached $1600 \mathrm{Oe}$. This range of the field can be used for tailoring the microwave properties of the two-dimensional arrays of nanosized stripes for applications in microwave filters. Also, the variation of the resonant frequency was accompanied by changes of the linewidth of the resonance in the range from 110 to $250 \mathrm{Oe}$. It is important to mention that the ferromagnetic resonance spectra have more than one resonant peak, but only the main peak was used to determine the angular dependence presented in Fig. 3. The additional resonant peaks are due to dimensional confinement of spin waves in the nanostructures, as reported in the works of others. ${ }^{10-12}$ However, the discussion of these magnetostatic modes is beyond the scope of this paper.

\section{CONCLUSIONS}

In spite of the presence of magnetostatic interactions and quantum confinement effects, the microwave properties of arrays of nanosized stripes of soft magnetic Py are dominated by their shape anisotropy, which is related to the aspect ratio of length to width of the stripes. For the arrays with large aspect ratios, the resonant field increased from about 800 to 2400 Oe when the applied field was rotated out of the easy direction in the plane of the array. This effect can be used for engineering the microwave properties of magnetic nanocomposites consisting of nanoparticles with different aspect ratios.

\section{ACKNOWLEDGMENTS}

This material is based on work supported by the National Science Foundation under NIRT Grant No. CCF0403673. The work was also partially supported by the LABoR Grant No. LEQSF(2005-06)-ENH-TR-99. Z.D., from Erskine College, Due West, SC, was sponsored through NSF Grant No. DMR-0243977.

${ }^{1}$ C. A. Ross et al., Phys. Rev. B 62, 14252 (2000)

${ }^{2}$ K. Nielsch, R. B. Wehrspohm, J. Barthel, J. Kirschner, U. Gösele, S. F. Fischer, and H. Kronmüller, Appl. Phys. Lett. 79, 1360 (2001).

${ }^{3}$ M. Vazquez, K. Pirota, M. Hernandez-Velez, Vm. Prida, D. Navas, R. Sanz, F. Batallan, and J. Velazquez, J. Appl. Phys. 95, 6642 (2004).

${ }^{4}$ B. Kuanr, L. Malkinski, R. E. Camley, Z. Celinski, and P. Kabos, J. Appl. Phys. 93, 8591 (2003)

${ }^{5}$ M. Vroubel, Y. Zhuang, B. Rejael, and J. N. Burghartz, J. Appl. Phys. 99, 08 P506 (2006).

${ }^{6}$ E. Salahun, P. Quéffélec, G. Tanné, A.-L. Adenot, and O. Acher, J. Appl. Phys. 91, 5449 (2002).

${ }^{7}$ C. Han, B. Y. Zong, P. Luo, and Y. H. Wu, J. Appl. Phys. 93, 9202 (2001).

${ }^{8}$ K. Nielsch, R. Hertel, R. B. Wehrspohn, J. Barthel, J. Kirchner, U. Gösele,

S. F. Fischer, and H. Kronmüller, IEEE Trans. Magn. 38, 2571 (2002).

${ }^{9}$ R. Hertel and J. Kirschner, Physica B 343, 206 (2004).

${ }^{10}$ S. O. Demokritov, B. Hillebrandts, and A. N. Slavin, IEEE Trans. Magn. 38, 2502 (2002).

${ }^{11}$ G. Gubbiotti, G. Carlotti, T. Okuno, T. Shinjo, F. Nizzoli, and R. Zivieri, Phys. Rev. B 69, 184409 (2003).

${ }^{12}$ R. Arias and D. L. Mills, Phys. Rev. B 70, 094414 (2004). 